\title{
Study of primary creep behavior of clayey soils in landslide area : suction effect
}

\section{Etude du comportement de fluage primaire des sols argileux dans une zone de glissement de terrain : Effet de la succion}

\author{
DAHHAOUI Hachimi ${ }^{1}$, BELAYACHI Naima ${ }^{2}$ etZADJAOUI Abdeldjalii ${ }^{1}$ \\ ${ }^{1}$ Laboratoire RISAM, Université AboubakrBelkaid.. BP 230 Rue Abi Ayad Abdelkrim. 13000. Tlemcen (Algérie) \\ ${ }^{2}$ Université d'Orléans, INSA-CVL, PRISME EA 4229, 8 Rue Léonard de Vinci, 45072 Orléans cedex 2, France
}

\begin{abstract}
Creep behavior of clayey soils plays an extremely important role in the landslide process. The soils that make up these sliding zones are often in unsaturated state. This point indicates the need to take into account the suction effect as hydric parameter on the long-term deformation of clayey soils. In this paper, a primary creep model named Modified Time Hardening (MTH) for unsaturated soils with different matric suction has been built. Based on the literature tests results[1][2], parameters $\mathrm{C} 1$ and $\mathrm{C} 2$ of the model have relations with suction and deviator stress level respectively. The primary creep strainwill be able to demonstrate unsaturated effect of the soils. comparison between the calculated results and the literature tests results shows a good coherence. The work underway at the university of Orleans will show later the relevance of model used in the present work.
\end{abstract}

Keywords:Creep behavior, Clay,Suction, Numerical modeling.

Résumé.Lecomportement en fluage des sols argileux joue un rôle essentiel dans le processus de glissement de terrain. Les sols constituants ces zones de glissements sont souvent à l'état non saturé, celaimplique la nécessité et l'importance de prendre en comptel'influence de lasuccion comme paramètre hydrique sur les déformations à long terme des sols argileux.Dans le présent article, un modèle de fluage primaire nommé Modified Time Hardening(MTH)a été considérépour étudier le comportement en fluage des sols non saturés. En se basant sur les résultats d'essais de la littérature[1][2], les deux paramètres $\mathrm{C} 1$ et $\mathrm{C} 2$ du modèle ont été modifiés en tenant en compte des relations en fonction de la succion et le niveau de déviateurrespectivement. La déformation de fluage primaire ainsi mesurée permettra de mettre en évidence l'effet de la non saturation des sols. La comparaison entre les résultats calculés et les résultats expérimentaux de la littérature montre une bonne cohérence. Les travaux en cours à l'université d'Orléans montrerons par la suite la pertinence de modèle utilisé dans le présent travail.

Mots Clés :Comportement en fluage, Argile, Succion, Modélisation.

\section{Introduction}

Ladépendance au temps des sols argileux est habituellement associéeà unfluage, c'est-à-dire un comportement sous des contraintes effectives constante, comme l'ont résumé Augustin et al. (2004)[3] et Lingaard et al. (2004)[4].les modèles de fluage peuvent être classés en troiscatégories Selon Purwodihardjo (2004) [5] : (empiriques, rhéologiques monodimensionnels et loi de comportement complètetridimensionnelle). La plupart des recherches scientifiques utilisent ces modèles dans des conditions de saturation complète, alors que l'état non saturé ne peut être négligé.Le présent article,se veut comme une contribution dans ce sens, c'est-à-dire présenteune étude du comportement au fluage d'un sol non saturé en considérant un modèle de fluage primaire. Dans un premier temps une revue de la littérature sur les modèles de fluage des sols non saturésa été présentée en détail. Dans un second temps le modèle proposé dans ce travail est détaillé. Il présente l'avantage d'être simple et utile dans la pratique de l'ingénieur. Ensuite, les résultats 
expérimentaux sur un solprovenant d'un grand glissement de terrain dans la région du réservoir des Trois Gorges en Chine ont été exploités pour valider notre modèle. Finalement, la mise en évidence de l'effet de la non saturation sur le fluage primaire a été discutés par les auteurs avec des pistes sur la nécessité d'amélioration des paramètres régissant le phénomène de fluage.

\section{Revue des travaux antérieurs}

Cette section, présente les modèles de fluage des sols argileux non saturés, développés dans un programme de suivi et d'auscultation d'un grand glissement de terrain dans la région du réservoir des Trois Gorges en Chine. Il s'agit d'un programme expérimental d'essais triaxiaux à succion et déviateur imposés, les auteursLai et al. (2010) [1] (2014) [6] et Zou et al. (2013) [2]ont réussi de prendre en considération la matrice de succion comme variable supplémentaire, favorisant l'amélioration des modèles de fluage classiques.

\subsection{Modèlesaméliorés par Lai et al. 2010 et 2014}

Les études effectuées par Lai et al. 2010[1] et 2014[6], porte sur l'amélioration du modèle de Singh-Mitchell (1968)[7] et le modèle de Mesri et al. (1981)[8]respectivement.

\subsubsection{Amélioration du modèle de Singh-Mitchell}

Le modèle de Singh et Mitchell (1968)[7]est une équation phénoménologique à trois paramètres, utilisé pour décrire le taux de déformation axiale des sols argileux soumis à un chargement constant. L'expression du modèle est composée d'une fonction exponentielle décrivant le chargement, et une fonction de puissance tenant en compte de l'effet du temps :

$\dot{\varepsilon}=A \cdot \exp \left(\alpha \cdot D^{r}\right) \cdot\left(\frac{t_{1}}{t}\right)^{m}$

Avec, $(\dot{\varepsilon})$ taux de déformation axial, $(t)$ le temps écoulé depuis l'application du chargement, $\left(t_{1}\right)$ le temps de référence désignant un stade précoce dans l'essai de fluage, $\left(D^{r}\right)$ niveau de déviateur désignant le rapport de la contrainte déviatorique imposée (q) sur la résistance au cisaillement induisant la rupture $\left(\mathrm{q}_{\mathrm{f}}\right),(A)(m) \operatorname{et}(\alpha)$ sont les paramètres de modèle.

L'intégration de l'équation 1 , lorsque $\mathrm{m} \neq 1$ produit une relation de type déformation-temps :

$\varepsilon=B \cdot \exp \left(\beta \cdot D^{r}\right) \cdot\left(\frac{t_{1}}{t}\right)^{\lambda}$

D'où, $(\varepsilon)$ déformation axial, $B=\frac{A \cdot t_{1}}{(1-m)}, \beta=\alpha, e t \lambda=1-m$.

Dans l'expression 2, la matrice de succion a été intégrée dans une nouvelle variablede niveau de déviateur $\left(D^{R}\right)$ définie comme suite :

$D^{R}=D^{r} \cdot\left(\frac{\sigma_{3}^{\prime}}{u_{a}}\right)$
Avec, $\left(\sigma_{3}^{\prime}\right)$ pression de confinement et $\left(u_{a}\right)$ pression de l'eau.

La relation entre la succion et le niveau de déviateur exprimé dans l'équation (3) a été justifié à travers l'analyse des résultats d'essais de fluage triaxiaux a succion contrôlée, dans lesquelles Lai et al. 2010[1]soulignent une relation d'inversement proportionnelle entre la déformation de fluage et la matrice de succion.

L'équation (4) ci-dessous présente l'amélioration du modèle de fluage établi par Lai et al. 2010 [1]

$\varepsilon=B \cdot \exp \left(\beta_{D R} \cdot D^{R}\right) \cdot\left(\frac{t}{t_{1}}\right)^{\lambda}$

Avec, $\left(\beta_{D R}\right)$ paramètre

correspondant au paramètre $(\beta)$ dans le cadre la nouvelle variabledéfinie $\operatorname{comme}\left(D^{R}\right)$.

Malgré les prévisions raisonnables pour la simulation de fluage des sols non saturés, les différences observées pour les niveaux de déviateursélevées suscitent les auteurs vers d'autres modèles et d'autres investigations.

\subsubsection{Amélioration du modèle de Mesri et al}

Le modèle constitutif de Mesri et al. (1981)[8] est une équation empirique, composée d'une fonction hyperbolique décrivant la relation contrainte-déformation et une fonction de puissance pour la relation déformationtemps :

$\varepsilon=\left[\frac{(\sigma 1-\sigma 3)_{f}}{E_{d}}\right]_{1} \frac{D_{1}}{1-\left(R_{f}\right)_{1} D_{1}}\left(\frac{t}{t_{1}}\right)^{n}$

Avec, $(\varepsilon)$ déformation axial, $(t)$ le temps écoulé depuis l'application $\mathrm{du}$ chargement, $\left(t_{1}=60\right.$ min $)$ temps de référence, $\left(\sigma_{1}-\sigma_{3}\right)_{f}$ la contrainte déviatorique maximal, $(D)$ niveau de déviateur désignant le rapport de la contrainte déviatorique (q)sur la résistance au cisaillement induisant la rupture $\left(\mathrm{q}_{\mathrm{f}}\right),\left(E_{d}\right)\left(R_{f}\right)$ et $(n)$ paramètres de fluage, $\left[\left(\sigma_{1}-\sigma_{3}\right)_{f}\right]_{1}$ et $\left(E_{d}\right)_{1}$ sont les valeurs $\operatorname{de}\left(\sigma_{1}-\sigma_{3}\right)_{f} \quad \operatorname{et}\left(E_{d}\right) \quad$ a l'instant $t=t_{l}$ respectivement, $(D)_{1}$ et $\left(R_{f}\right)_{1}$ sont le niveau de déviateur $(D)$ et le paramètre $\left(R_{f}\right)$ au même instant.

Selon Mesri et al. (1981)[8], les trois paramètres $\left(E_{d}\right)(R f)$ et $(n)$ dans l'équation (5)peuvent être exprimés en terme de $\frac{\left(\sigma_{1}-\sigma_{3}\right) f}{E_{d}}$ et $\varepsilon_{f}$, d'où Lai et al. (2014)[6] a déduit que la matrice de succion peut avoirun effet $\operatorname{sur} \frac{\left(\sigma_{1}-\sigma_{3}\right)_{f}}{E_{d}}$ et $\varepsilon_{f}$,cela lui avait permis d'établir une relation entre la matrice de succion et le paramètre $R_{f}$ :

$R_{f}=a \cdot\left(\frac{s}{p_{a}}\right)+b$ 


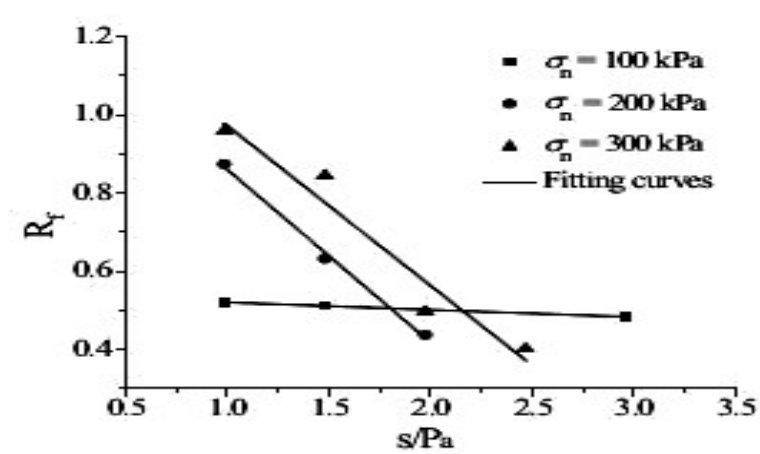

Fig. 1. Relation entre le paramètre $\mathrm{R}_{\mathrm{f}}$ et le rapports/ $\mathrm{Pa}[6]$.

Avec, $(P a) \quad$ pression atmosphérique(101,33 $\mathrm{kPa}) ;(a)$ et $(b)$ valeurs constantes obtenue à partir des courbes linéaires illustrés sur la "figure 1".

L'équation (7) présente le nouveau modèle empirique de Lai et al. (2014)[6], pour les argiles non saturées.Ce dernier prend en considération l'effet de la succion sur le comportement en fluage de l'argile étudiée, il s'adapte de manière satisfaisante avec les résultats expérimentaux.

$\varepsilon=\left[\frac{\left(\sigma_{1}-\sigma_{3}\right)_{f}}{E_{d}}\right] \frac{D_{1}}{1-[a(s / P a)+b] D_{1}}\left(\frac{t}{t_{1}}\right)^{n}$

Il convient de mentionner qu'en raison des résultats de tests limités, l'influence de la pression de confinement sur le comportement de fluage de l'argile étudiée n'été pas pris en compte, ce qui est l'une des principales limites de ce modèle.

\subsection{Modèle amélioré par Zou et al. 2013}

Le modèle de fluage de Burgers,est un modèle rhéologique composé d'un corps de Maxwell relié en série avec un corps de Kelvin "figure 2".

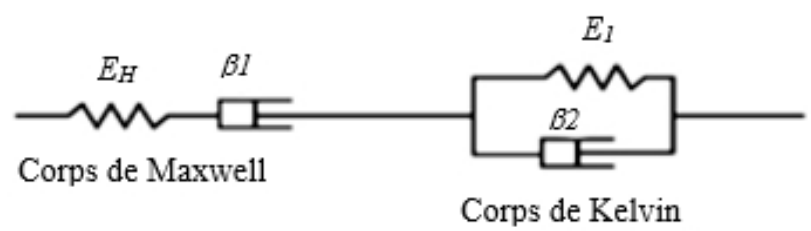

Fig. 2. Modèle de fluage de Burgers[2]

Le travail de Zou et al.2013 [2] porte sur l'amélioration du modèle étendu de Burgers. Ce dernier est développé en insérant un autre corps de kelvin dans le modèle de

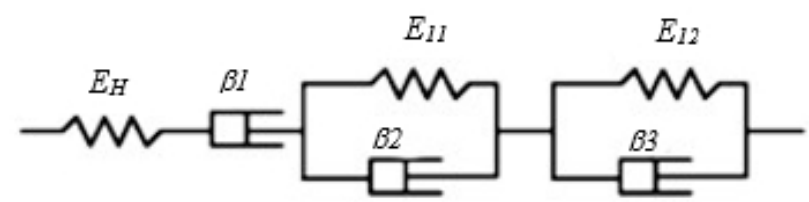

Burgers "figure 3".

Fig. 3. Modèle de fluage « Burgers étendu » $(\mathrm{M}-2 \mathrm{~K})[2]$

L'équation constitutive de ce modèlepeut être décrite selon Sun. 1999[9]comme: $\varepsilon(t)=\sigma \cdot\left\{\frac{1}{E H}+\frac{1}{\beta 1}+\frac{1}{E 11}\left[1-\exp \left(-\frac{E 11 . t}{\beta 2}\right)\right]+\right.$

$\left.\frac{1}{E 12}\left[1-\exp \left(-\frac{E 12 . t}{\beta 3}\right)\right]\right\}$

Avec, $\left(E_{H}\right)\left(E_{11}\right)$ et $\left(E_{12}\right)$ modulesélastiques du corps de Maxwell et les corps de Kelvin respectivement, $(\beta 1)(\beta 2)$ et $(\beta 3)$ présentent la viscosité du corps de Maxwell et les corps de Kelvin respectivement, $(t)$ temps, $(\sigma)$ contrainte.

A partir des hypothèses de Yan et al.2010[10] l'équation (8) été transformaità un état tridimensionnel pour prendre en compte la matrice de succion dans le modèle :

$\varepsilon_{i j}=\frac{S^{\prime}{ }_{i j}}{3 K}+\frac{S^{\prime \prime}{ }_{i j}}{3 G H}+\frac{S^{\prime \prime}{ }_{i j}}{3 \eta 1} t+\frac{S^{\prime \prime}{ }_{i j}}{3 G 11} \cdot\left[1-\exp \left(\frac{-G 11 . t}{\eta 2}\right)\right]+$

$\frac{S^{\prime \prime} i j}{3 G 12}\left[1-\exp \left(\frac{-G 12 . t}{\eta 3}\right)\right]$

Avec, $\left(s_{i j}^{\prime}\right)$ tenseur de contrainte sphérique, $\left(s_{i j}^{\prime \prime}\right)$ tenseur de contrainte déviatorique, $\left(G_{H}\right)\left(G_{11}\right)$ et $\left(G_{12}\right)$ modules de cisaillement du corps de Maxwell et les corps de Kelvin respectivement, $\left(\eta_{1}\right)\left(\eta_{2}\right)$ et $\left(\eta_{3}\right)$ les coefficients visqueux du corps de Maxwell et les corps de Kelvin respectivement, $(K)$ module d'élasticité isotrope déterminé par l'équation (10) :

$K=\frac{2 G_{H}(1+v)}{3(1-2 v)}$

Avec, $(v)$ coefficient de poisson.

Des relations linéaires ont été trouvé entre les paramètres de l'équation (9) et la succion. Le nouveau modèle de fluage obtenu est comme suit :

$\varepsilon_{i j}=\frac{S_{i j}^{\prime}}{3 K^{\prime}}+\frac{S^{\prime \prime}{ }_{i j}}{3 G^{\prime} H}+\frac{S^{\prime \prime}{ }_{i j}}{3 \eta^{\prime} 1} t+\frac{S^{\prime \prime}{ }_{i j}}{3 G^{\prime} 11}\left[1-\exp \left(\frac{-G^{\prime} 11 . t}{\eta^{\prime} 2}\right)\right]+$

$\frac{S^{\prime \prime}{ }_{i j}}{3 G^{\prime} 12}\left[1-\exp \left(\frac{-G^{\prime} 12 . t}{\eta^{\prime} 3}\right)\right]$

L'équation (11) présente le modèle de fluage amélioré par Zou et al.2013[2], les nouveaux paramètres $\left(G_{H}^{\prime}\right)\left(G_{11}^{\prime}\right)\left(G_{12}^{\prime}\right)\left(\eta_{1}^{\prime}\right)\left(\eta_{2}^{\prime}\right)$ et $\left(\eta_{3}^{\prime}\right)$ prennent en compte la matrice de succion dans le modèle. La comparaison entre la simulation et l'expérimentation a montré l'efficacité et la capacité prédictive de ce dernier. En outre, même si le modèle prend en considération une large gamme de succion comprise entre 50 et $300 \mathrm{kPa}$, il reste limité à un seul niveau de déviateur maintenu à 0,55 contrairement au modèles précédents Lai et al. 2010[1]et 2014[6]. Cela, implique la nécessité d'autres essais de fluage a succion contrôlée et à différents niveaux de contraintesdéviatorique.

\section{Comportement en fluagedes sols non saturés : Proposition d'un modèle}

Dans cette section, le modèle de fluage primaire « Modified Time Hardening »(MTH)[11] est présenté. Ensuite une relation est établie entre l'un des paramètres $\mathrm{du}$ modèle et la succion, puis un second paramètreest induit avec le niveau de déviateur appliqué. Sur cette base, le modèle est reconsidéré pour calculer la 
déformation en fluage en prenant en compte la succion et le niveau du déviateur.

\subsection{Modèle de fluage primaire «Modified Time Hardening »[11]}

Comme décrit dans la section 2, les modèles développés font déjà preuve d'une bonne efficacité, néanmoins leurs présences dans un code de calculs aux éléments finis n'est pas toujours évidente. Cela implique une procédure d'implémentation qui requiert des connaissances sur le langage de programmation du logiciel utilisé.

Dans ce travail, l'équation de fluage primaire du logiciel ANSYS,intitulé« Modified Time Hardening» (MTH)a été sélectionnée. Le choix de ce modèle est justifié par sa capacité et sa performance pour la représentation de fluage primaire considéré comme réponse macroscopique lors d'un essai de fluage triaxial[12].

L'expression générale du modèle est comme suite :

$\varepsilon_{c r}(t)=\left(\frac{C 1}{C 3+1}\right) \cdot q^{C 2} \cdot t^{C 3+1}$

Avec, $\left(\varepsilon_{c r}\right)$ ladéformation de fluage, $(q)$ lacontrainte déviatorique, $(t)$ est letemps du chargement et $\mathrm{Ci} \quad(i=1$ 3)paramètres de fluage.

\subsection{Prise en compte de la succion}

Pour l'élaboration du modèle de fluage de l'argile non saturé, une méthode alternative consiste d'abord à analyser la prédiction du modèle sur les résultats d'essais de fluage triaxiaux menée par Zou el al[2]. Les tests ont été réalisés sous une pression de confinement $\sigma_{3}^{\prime}$ maintenu à $100 \mathrm{kPa}$, et un niveau de déviateur $D^{r}$ maintenu à 0,55 .Un jeu de paramètres pour chacun des essais est répertorié dans le Tableau 1.L'identification des paramètres est réalisée par minimisation de l'écart entre le résultat du calcul numérique et la courbe expérimentale.

Tableau 1.Paramètres du modèle en fonction de la succion

\begin{tabular}{|c|c|c|c|c|c|}
\hline $\mathbf{s}(\mathbf{k P a})$ & $\mathbf{q}(\mathbf{k P a})$ & $\mathbf{D}^{\mathbf{r}}$ & $\mathbf{C 1}$ & $\mathbf{C 2}$ & $\mathbf{C 3}$ \\
\hline 50 & 103 & 0,55 & $9,5.10^{-5}$ & 1,64 & 0,075 \\
\hline 100 & 124 & 0,55 & $6,8.10^{-5}$ & 1,64 & 0,075 \\
\hline 150 & 141 & 0,55 & $5,3.10^{-5}$ & 1,64 & 0,075 \\
\hline 200 & 188 & 0,55 & $3,4.10^{-5}$ & 1,64 & 0,075 \\
\hline 250 & 191 & 0,55 & $2.9 .10^{-5}$ & 1,64 & 0,075 \\
\hline
\end{tabular}

Sur la base des résultats du Tableau 1, l'augmentation de la succion conduit à la diminution du paramètre $\mathrm{C} 1$ d'où le choix d'une relation logarithmique entre $\mathrm{C} 1-\mathrm{s} / \mathrm{Pa}$ "figure 4".

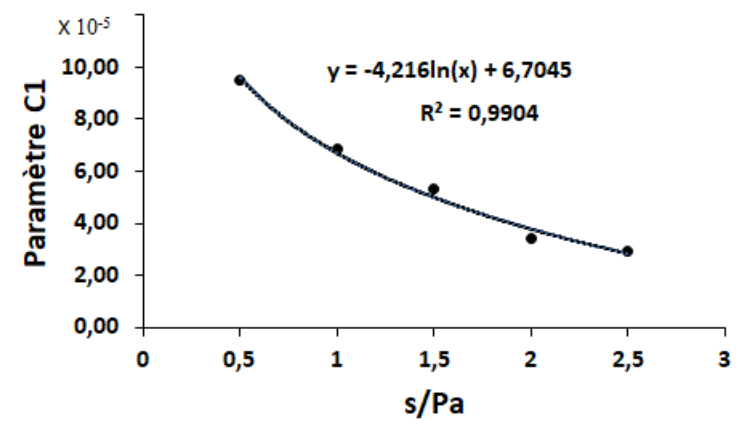

Fig. 4. Relation entre le paramètre $\mathrm{C} 1$ et le rapports/Pa.

Cependant, la prise en compte du niveau de déviateur $\left(D^{r}\right)$ dans le modèle ne peut être négligé. Dans le Tableau 2, les paramètres du modèle ont été déterminés à partir des résultats d'essais menée par Lai et al. (2010)[1] sous une pression de confinement $\sigma^{\prime} 3$ maintenu à 100 $\mathrm{kPa}$, et des niveaux du déviateur $\left(D^{r}\right)$ différents. L'analyse des résultats, montre la sensibilité du paramètre $\mathrm{C} 2$ vis-àvis leniveau du déviateur.Une relation de puissance a été établie entre C2- $\mathrm{D}^{\mathrm{r}}$ "figure 5". En revanche,le paramètre C3 reste constant, ildépend du type de sol et de sa composition.Il vaut 0,075 pourleséchantillons d'argile étudiées.

Tableau 2. Paramètres du modèle pour différents niveaux du déviateur

\begin{tabular}{|c|c|c|c|c|c|}
\hline $\mathbf{D}^{\mathbf{r}}$ & $\mathbf{q}(\mathbf{K P a})$ & $\mathbf{S}(\mathbf{k P a})$ & $\mathbf{C 1}$ & $\mathbf{C 2}$ & $\mathbf{C 3}$ \\
\hline 0,112 & 39,2 & 250 & $2,9.10^{-5}$ & 1,04 & 0,075 \\
\hline 0,161 & 54,9 & 200 & $3,4.10^{-5}$ & 1,37 & 0,075 \\
\hline 0,170 & 62,9 & 300 & $2,1.10^{-5}$ & 1,28 & 0,075 \\
\hline 0,258 & 90,2 & 250 & $2,9.10^{-5}$ & 1,30 & 0,075 \\
\hline 0,323 & 109,8 & 200 & $3,4.10^{-5}$ & 1,56 & 0,075 \\
\hline 0,340 & 125,8 & 300 & $2,1.10^{-5}$ & 1,53 & 0,075 \\
\hline 0,403 & 141,2 & 250 & $2,9.10^{-5}$ & 1,45 & 0,075 \\
\hline 0,438 & 149 & 200 & $3,4.10^{-5}$ & 1,63 & 0,075 \\
\hline 0,510 & 188,7 & 300 & $2,1.10^{-5}$ & 1,77 & 0,075 \\
\hline 0,546 & 191,2 & 250 & $2,9.10^{-5}$ & 1,58 & 0,075 \\
\hline 0,553 & 188,2 & 200 & $3,4.10^{-5}$ & 1,67 & 0,075 \\
\hline 0,669 & 227,4 & 200 & $3,4.10^{-5}$ & 1,76 & 0,075 \\
\hline 0,680 & 251,6 & 300 & $2,1.10^{-5}$ & 1,90 & 0,075 \\
\hline 0,784 & 266,6 & 200 & $3,4.10^{-5}$ & 1,80 & 0,075 \\
\hline
\end{tabular}

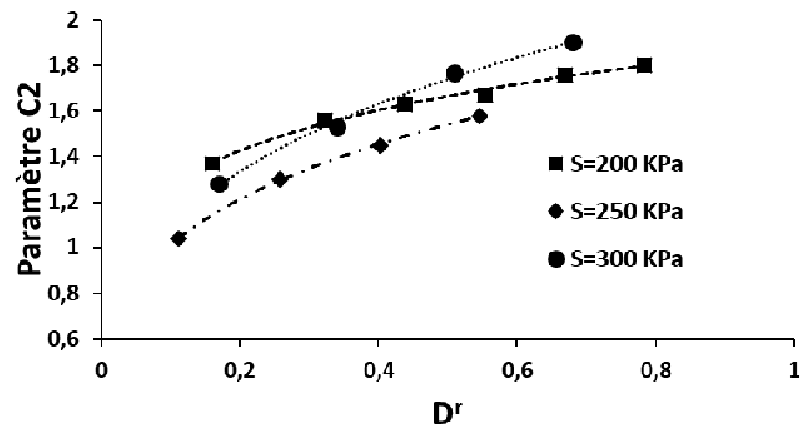

Fig. 5.Relation entre le paramètre $\mathrm{C} 2$ et le niveau de déviateur $\mathrm{D}^{\mathrm{r}}$

Compte tenu de la variation du paramètre $\mathrm{C} 1$ en fonction de la succion "figure 4", et la variation du paramètre $\mathrm{C} 2$ en fonction du niveau de déviateur appliqué "figure 5", le modèle est reconsidéré comme suit :

$\varepsilon_{c r}(t)=\left(\frac{C_{1}^{\prime}}{1,075}\right) \cdot q^{C_{2}^{\prime}} \cdot t^{1,075}$ 
Le calcul des nouveaux paramètres dans l'équation (13) est illustré dans le Tableau 3.

Tableau 3.Conditions et paramètresdu modèle amélioré

\begin{tabular}{|c|c|}
\hline Conditions hydriques & Paramètres \\
\hline Succion $[50-250] \mathrm{kPa}$ & $c^{\prime}{ }_{1}=-4.10^{-5} \ln (\mathrm{S} / \mathrm{Pa})+7.10^{-5}$ \\
\hline Succion $[200] \mathrm{kPa}$ & ${c^{\prime}}_{2}=1,87 . D^{r, 17}$ \\
\hline Succion $[250] \mathrm{kPa}$ & $c^{\prime}{ }_{2}=1,84 . D^{r 0,26}$ \\
\hline Succion $[300] \mathrm{kPa}$ & $c^{\prime}{ }_{2}=2,12 . D^{r 0,29}$ \\
\hline
\end{tabular}

\subsection{Validation}

Le but de cette section se veut comme une évaluation du modèle avec la prise en compte des paramètres avec les relations présentées ci-dessus. A partir des conditions illustrées dans le Tableau 3,la matrice de succion et le niveau de déviateur ont été pris en compte pour calculer la déformation de fluage. Les résultats d'essais de fluage triaxiaux publié dans le travail de Lai et al. 2010 [1]ont été simulés, la comparaison entre les résultats montre la capacité prédictive du modèle pour le comportement au fluage des sols non saturés "figure 6".

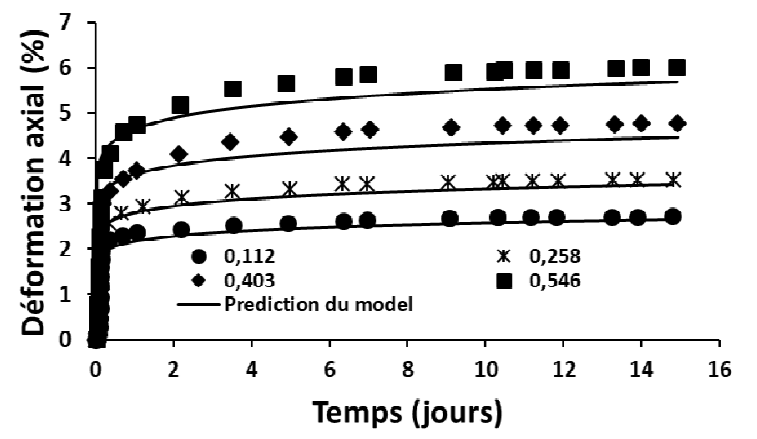

Fig. 6.Simulations d'essais de fluage triaxiaux à succion $\mathrm{s}=250$ $\mathrm{kPa}$ et différents niveaux de contrainte déviatorique

Le modèle est ensuite positionné vis-à-vis les modèles qui ont été présentés dans la section 2.La comparaison illustrée dans la "figure 7"montre une capacité prédictive presque identique entre les différents modèles.

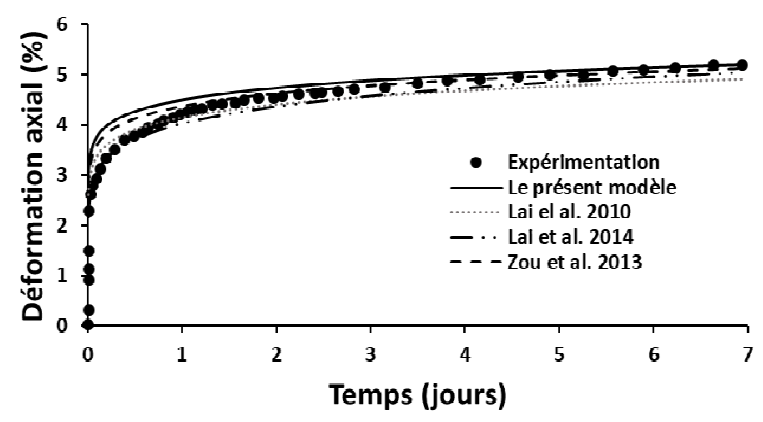

Fig. 7.Simulation d'essai de fluage triaxial à succion $s=200 \mathrm{kPa}$ et niveau de contrainte déviatorique $\mathrm{D}=0.55$

Il convient de mentionner qu'en raison du manque des résultats pour d'autres valeurs de succion, le modèle est limité à prédire le fluage pour une gamme de succion comprise entre 200 et $300 \mathrm{kPa}$. Il convient également de souligner que le modèle est limité à des déformations axiales et ne peut prendre en compte la déformation latérale ou de cisaillement.

\section{Conclusion}

Un modèle de fluage primaire pour les sols non saturés simulant des tests de fluage triaxiaux a été considéré avec des relations interprétant la sensibilité des paramètres à la succion et au niveau de contrainte. Basé sur une relation logarithmique trouvée entre le paramètre $\mathrm{C} 1$ et la succion, et une relation de puissance entre le paramètre $\mathrm{C} 2$ et le niveau de déviateur, le modèle prend en compte l'effet dela non saturation et une large gamme de niveau de déviateur, avec des coefficients de corrélation supérieurs à 0,98 . Les résultats simulés sont en accord avec les résultats expérimentaux.

L'avantage d'amélioration de ce modèledisponible dans un code d'éléments finis ANSYS, permettra une application étendu pour la simulation du phénomène sous la fluctuation du niveau d'eau cyclique, pour une période de plus long terme. Ceci, permet une base importante pour la prédiction et l'évaluation des glissements de terrain, dansles futures investigations numériques.

\section{Références bibliographiques}

1. X. Lai, S. Wang, H. Qin, and X. Liu, "Unsaturated creep tests and empirical models for sliding zone soils of Qianjiangping landslide in the Three Gorges," J. Rock Mech. Geotech. Eng., vol. 2, no. 2, pp. 149-154, (2010).

2. L. Zou, S. Wang, and X. Lai, "Creep model for unsaturated soils in sliding zone of Qianjiangping landslide," J. Rock Mech. Geotech. Eng., vol. 5, no. 2, pp. 162-167, Apr.(2013).

3. A. Augustesen, M. Liingaard, P. V Lade, and M. Asce, "Evaluation of Time-Dependent Behavior of Soils," Int. J. Geomech., vol. 4, no. 3, pp. 137-156, (2004).

4. M. Liingaard, A. Augustesen, P. V Lade, and M. Asce, "Characterization of Models for TimeDependent Behavior of Soils," Int. J. Geomech., vol. 4, no. 3, pp. 157-177, (2004).

5. A. Purwodihardjo, "Modélisation des déformations différées lors du creusement des tunnels," Ecole centrale de Lyon, (2004).

6. X. Lai, S. M. Wang, W. M. Ye, and Y. J. Cui, "Experimental investigation on the creep behavior of an unsaturated clay," Can. Geotech. J., vol. 51, no. 6, pp. 621-628, (2014).

7. A. Singh and J. K. Mitchell, "General stress-straintime function for soils," J. Soil Mech. Found Eng. Div. ASCE, vol. 94, no. 1, pp. 21-46, (1968).

8. G. Mesri, E. Febres-Cordero, D. Shields, and A. Castro, "Shear stress-strain-time behaviour of clays," Geotechnique, vol. 31, no. 4, pp. 537-552, (1981).

9. J. Sun, "Rheological of geotechnical materials and its application to engineering," China Architecture and Building Industry Press, (1999). 
10. Y. A. N. Yan, W. A. N. G. Si-jing, and W. A. N. G. En-zhi, "Creep equation of variable parameters based on Nishihara model," Rock Soil Mech., vol. 31, no. 10, pp. 3025-3035, (2010).

11. ANSYS, "ANSYS Mechanical APDL Theory Reference," ANSYS Inc, vol. Release15, no. November, pp. 1-909, (2013).

12. R. Plassart, "Modélisation hydromécanique du comportement des ouvrages souterrains avec un modèle élastoviscoplastique," Dr. Diss. Vandoeuvreles-Nancy, INPL, (2011). 